\title{
CONSTRAINED TOPOLOGY OPTIMIZATION FOR ADDITIVE MANUFACTURING OF STRUCTURAL COMPONENTS IN ANSYS ${ }^{\circledR}$
}

\author{
Davin Jankovics, Hossein Gohari, Ahmad Barari \\ Faculty of Engineering and Applied Science \\ University of Ontario Institute of Technology \\ Oshawa, Ontario \\ davin.jankovics@uoit.ca, hossein.goharibahabadi@uoit.ca, ahmad.barari@uoit.ca
}

\begin{abstract}
Topology Optimization is currently the main technique to optimize an objects structural design. This method commonly produces parts that have exceedingly complex geometry. Additive manufacturing (AM) is the main manufacturing process to produce these optimized designs due to the flexibility and speed it offers. However, results of topology optimization without considering manufacturing process limits, even AM ones, may result in designs that are expensive and difficult to build. This paper presents a topology optimization filter that minimizes the effect of overhang structures. These structures are very difficult to manufacture using conventional AM techniques. In order to constrain the gradient compliances with respect to densities and converge the results towards a structure with the least amount of overhang structures, sensitivities are modified using the proposed filter. To implement the proposed filter and the base topology optimization methods ESO and SIMP, ANSYS Parametric Design Language (APDL) is employed within the ANSYS ${ }^{\circledR}$ Workbench $^{\mathrm{TM}}$ environment. The results of a case study using the different topology optimization methods are investigated. Finally, an implementation of the proposed AM filter is used to solve an MBB-beam problem. The result is a structure that needs the least amount of support structure.
\end{abstract}

Keywords-Additive Manufacturing; 3D Printing; Topology Optimization; Finite Element Analysis; ANSYS ${ }^{\circledR}$; ANSYS Parametric Design Language (APDL)

\section{INTRODUCTION}

Topology optimization is a subset of structural optimization with the goal of finding an optimal design for a certain set of constraints. It can be described as an iterative process where the results of a finite element analysis (FEA) are used to determine the optimal geometry and locations of voids in a solid object [1].

In the context of engineering, finite element analysis is a mathematical method of solving a system which cannot easily be described using closed form equations. Instead it breaks the system into smaller, easier to solve or approximate, parts using what are known as elements [2]. One popular FEA software for engineering is ANSYS $^{\circledR}$. Released in 1971 and still being actively developed, ANSYS ${ }^{\circledR}$ can be used to solve a diverse field of engineering problems including static structural, dynamic, heat transfer, fluid flow, and electromagnetism. Currently, the user facing software of ANSYS ${ }^{\circledR}$ is Workbench ${ }^{\mathrm{TM}}$, from which a coding language called ANSYS Parametric Design Language (APDL) is called when simulations are run. This language executes the FEA, calculating all necessary parameters to solve the analysis. For advanced users, APDL is user accessible through custom command scripts that can be injected at any point during the analysis.

Creating a topology optimizer from scratch would include the development of a topology optimization algorithm and the development of a complete FEA solver. Additionally, if the creation and editing of geometry was also required, a computer aided design software would also be required. Instead, the accessible interface of ANSYS ${ }^{\circledR}$ and its mature FEA solver can be utilized to create a more extensive and easy to use topology optimizer through custom injected APDL scripts. Since ANSYS $^{\circledR}$ includes Multiphysics simulations, the ability to add additional constraints and objectives, including manufacturing constraints and coupled analyses may become possible.

Additive manufacturing (AM) is a fairly young and quickly developing technology that overcomes many traditional manufacturing limits for complex geometries. However, all additive manufacturing methods still have some constraints that need to be considered during the design process. The use of disposable support material is one method to work around these constraints, but it is not a perfect solution as it often leads to a poor surface quality finish [3]. This support material is used to prevent failure during the manufacturing procedure by preventing overhang structures in the part geometry from collapsing, or otherwise ruining the final parts finish [4]. Since most AM processes use a manufacturing method where material is added layer by layer, each being fused to the previous, it can be said that there exists an angle where supports will be required.

Other approaches to minimize the prevalence of overhangs in topology optimized parts have been developed [5]. In this paper, a 3D implementation of the evolutionary structural optimization (ESO) and solid isotropic material with penalisation (SIMP) methods are presented along with a new 
filtering method to reduce the amount of material needed for supports, all in ANSYS ${ }^{\circledR}$.

\section{BACKGROUND}

\section{A. Evolutionary Structural Optimization (ESO) Method}

A topology optimization problem seeks to minimize compliance while satisfying various constraints such as a given amount of material, weight, manufacturing requirement, and cost [1]. The measure of stiffness of a structure can be calculated using a finite element analysis. In literature, instead of stiffness, mean compliance $\mathrm{C}$, is considered as the objective for topology optimization. The evolutionary procedure based on stiffness as the objective function can be described as follows:

1) Discretize the body for FEA.

2) FEA determines sensitivity number for each element.

3) Remove elements with lowest sensitivity number based on element removal ratio.

4) Repeat the procedure to reach termination term (maximum displacement, stress, etc).

\section{B. Solid Isotropic Material with Penalization (SIMP)}

Minimizing compliance is the most used objective in topology optimization, shown in (1).

$$
\begin{gathered}
\min _{x}: c(\rho)=U^{T} K U=\sum_{e=1}^{N}\left(\rho^{e}\right)^{p} u_{e}^{T} k^{e} u_{e} \\
\text { Subject to: } \quad \frac{V(\rho)}{V_{0}}=f \\
; K U=F \\
; 0<\rho_{\text {min }} \leq x \leq 1
\end{gathered}
$$

Where the stiffness matrix for each element can be found from (2).

$$
\begin{gathered}
k^{e}=\int_{\Omega} H^{T} D H d \Omega \\
=\sum_{i=1}^{2} \sum_{j=1}^{2} w_{i} w_{j}|J(\xi, \eta)| H(\xi, \eta)^{T} D H(\xi, \eta)
\end{gathered}
$$

Here, the starting Young's modulus of an object does not influence the optimal results of topology optimization, since any changes will be relative. The general solution of the topology optimization is constructed to minimize compliance using a Lagrange multiplier method as shown in (3).

$$
L(\rho, \lambda)=f^{T} d(\rho)+\lambda^{T}(f-K(\rho) d(\rho))
$$

By setting $\lambda=d$ and $k^{e}=\int_{\Omega} H^{T}\left(E_{0}+\left(\rho^{e}\right)^{p} E_{1} D^{*} H d \Omega\right.$, the derivative of the lagrangian equation with respect to the design variables can be determined as (4) [6].

$$
\frac{\partial L(\rho, \lambda)}{\partial \rho^{e}}=-P\left(\rho^{e}\right)^{p-1} d^{e T} K_{f}^{e} d^{e}
$$

Updating of element densities heuristically can be achieved using the bi-section algorithm. A heuristic algorithm is used here based on the Optimality Criteria (OC) method shown in (5) [7]. A numerical constraint has been considered to limit the update for densities, $m$.

$$
\rho_{\text {new }}^{e}=\left\{\begin{array}{c}
\text { if } \rho^{e} B_{e}^{\eta} \leq \max \left(\rho_{\min }, \rho^{e}-m\right) \\
\max \left(\boldsymbol{\rho}_{\min }, \boldsymbol{\rho}^{\boldsymbol{e}}-\boldsymbol{m}\right) \\
\text { if } \max \left(\rho_{\min }, \rho^{e}-m\right)<\rho^{e} B_{e}^{\eta}<\min \left(1, \rho^{e}+m\right) \\
\boldsymbol{\rho}^{e} \boldsymbol{B}_{\boldsymbol{e}}^{\eta} \\
\text { if } \min \left(1, \rho^{e}+m\right) \leq \rho^{e} B_{e}^{\eta} \\
\min \left(\mathbf{1}, \boldsymbol{\rho}^{e}+\boldsymbol{m}\right)
\end{array}\right\}
$$

Where $B_{e}^{\eta}=-\frac{1}{\lambda} \frac{\partial c}{\partial \rho^{e}}$ and $\eta(=1 / 2)$ is a numerical damping coefficient.

\section{Implementation of Overhang Constraint}

Applications of developed topology optimization methods have been very restricted due to the limitations in manufacturing and inspection of the resulting complex geometries. A successful design process needs to be conducted by considering all of the manufacturing and inspection requirements [8]. Although additive manufacturing processes are still not able to produce high surface qualities [9], [10], they are highly flexible in producing the complex geometries produced by topology optimization [11], [12]. Also, the advances in coordinate metrology algorithms allow relatively fast inspection of the complex topologies resulting by the topologically optimized designs [13].

These normal topology optimization methods converge to a computationally stiff structure, but without regard for the difficulty of manufacturing. Here is where another constraint is added that limits the surface area of elements to be less than a specified value for the optimal solution. This approach can be used to implement an overhang constraint. In the methodology section a new scheme is developed to estimate the sensitivities of overhanging elements in 3D space. These elements can then be penalized with respect to their overall sensitivity.

\section{Using APDL in ANSYS ${ }^{\circledR}$}

All the simulation and FEA functionality of ANSYS $^{\circledR}$ comes from APDL commands [2]. Any FEA parameter that is calculated in $\mathrm{ANSYS}^{\circledR}$ can be read, and as logic applies, can be modified.

The syntax for an APDL commands starts with the name of the commands, followed by a comma, and then the operators of the commands, all separated by commas. There are also logical operators such as do, if, ifelse, and while.

The actual FEA solver in ANSYS ${ }^{\circledR}$ operates much like other FEA solvers [14]. There are three overall levels within the solver, the first being the preprocessor. This is where the mesh of the geometry is defined, as well as the loads on the body and boundary conditions. Next, the solver processes the generated simultaneous equations, which results in the values for degrees of freedom for the nodes on each element. This data is then taken into the final level, the postprocessor, where all other results are 
calculated, such as stress and strain. It is important to note that only certain APDL commands can be executed in certain levels of the FEA, limiting how one can interact with the program. However, with a large selection of pre-defined commands combined with logical operators, essentially anything related to the FEA process can be modified and manipulated.

\section{MethODOLOGY}

\section{A. Implementing the Topology Optimization Overhang Constraint}

Using a similar method to that Sigmund used to solve the optimality criteria, the area of an element can be considered as a new constraint. A method has been developed to calculate the derivative of the surface area of each element with respect to its density [15]. Using the same approach shown in (6), the approximate surface area sensitives are calculated for the bottom elements, which are multiplied by a constant coefficient $\gamma$, as shown in Fig. 1.

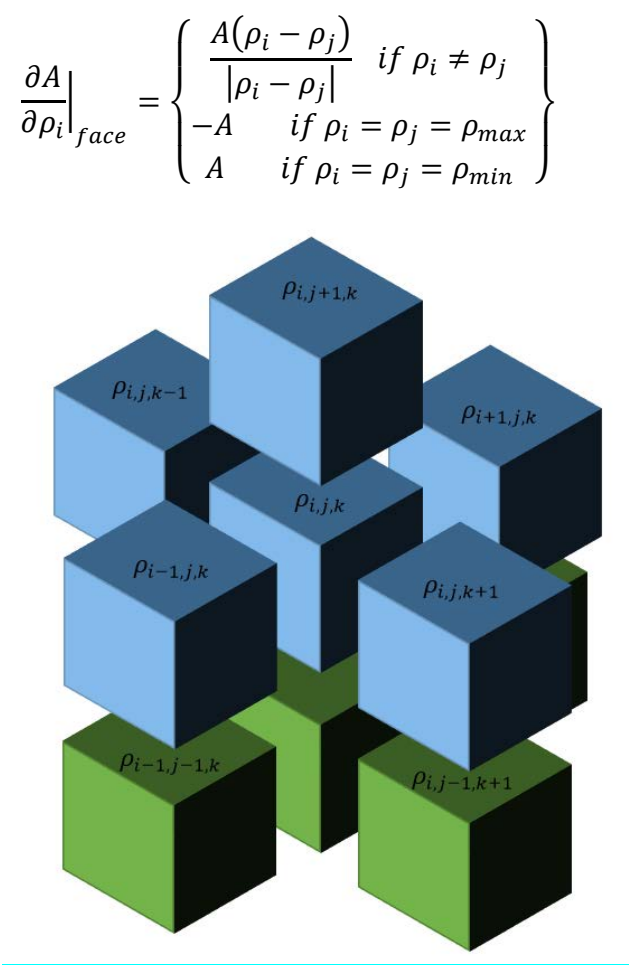

Figure 1. Elements that are considered in the modification of perimeter sensitives based on each element connectivity

For the estimation of the overhang sensitivities, a scheme used to penalize overhang structures shown in (7) is developed. Individual element connectivity's are found using a predefined function inside ANSYS $^{\circledR}$ that finds the six elements connected to the element.

$$
\begin{gathered}
\frac{\partial \mathrm{A}}{\partial \rho^{e}}=\left\{\left.\frac{\partial \mathrm{A}}{\partial \rho_{\mathrm{i}}}\right|_{\mathrm{face}(i, j+1, k)}+\left.\frac{\partial \mathrm{A}}{\partial \rho_{\mathrm{i}}}\right|_{\mathrm{face}(i-1, j, k)}+\left.\frac{\partial \mathrm{A}}{\partial \rho_{\mathrm{i}}}\right|_{\mathrm{face}(i-1, j, k+1)}+\right. \\
\left.\frac{\partial \mathrm{A}}{\partial \rho_{\mathrm{i}}}\right|_{\mathrm{face}(i+1, j, k)}+\gamma\left[\left.\frac{\partial \mathrm{A}}{\partial \rho_{\mathrm{i}}}\right|_{\mathrm{face}(i-1, j-1, k)}+\right. \\
\left.\frac{\partial \mathrm{A}}{\partial \rho_{\mathrm{i}}}\right|_{\mathrm{face}(i-1, j, k-1)}+\left.\frac{\partial \mathrm{A}}{\partial \rho_{\mathrm{i}}}\right|_{\mathrm{face}(i+1, j-1, k)}+ \\
\left.\left.\left.\frac{\partial \mathrm{A}}{\partial \rho_{\mathrm{i}}}\right|_{\mathrm{face}(i, j-1, k)}+\left.\frac{\partial \mathrm{A}}{\partial \rho_{\mathrm{i}}}\right|_{\mathrm{face}(i, j-1, k-1)}\right]\right\}
\end{gathered}
$$

From this, the modified sensitivities are shown in (8).

$$
C_{e}^{\eta}=-\frac{1}{\lambda}\left[\frac{\partial c}{\partial \rho^{e}}+\delta \frac{\partial A}{\partial \rho^{e}}\right]
$$

Now, the update scheme shown in (5) is used for the topology optimization procedure, except the modified sensitivities are used instead of the compliance sensitivities to converge the densities away from overhang structures, (9).

$$
\rho_{n e w}^{e}=\left\{\begin{array}{c}
\text { if } \rho^{e} C_{e}^{\eta} \leq \max \left(\rho_{\min }, \rho^{e}-m\right) \\
\boldsymbol{m a x}\left(\boldsymbol{\rho}_{\min }, \boldsymbol{\rho}^{\boldsymbol{e}}-\boldsymbol{m}\right) \\
\text { if } \max \left(\rho_{\min }, \rho^{e}-m\right)<\rho^{e} C_{e}^{\eta}<\min \left(1, \rho^{e}+m\right) \\
\boldsymbol{\rho}^{e} \boldsymbol{C}_{\boldsymbol{e}}^{\eta} \\
\text { if } \min \left(1, \rho^{e}+m\right) \leq \rho^{e} C_{e}^{\eta} \\
\min \left(\mathbf{1}, \boldsymbol{\rho}^{\boldsymbol{e}}+\boldsymbol{m}\right)
\end{array}\right\}
$$

\section{B. Topology Optimization using APDL}

Building the topology optimizer on top of ANSYS ${ }^{\circledR}$ allows for a more flexible design platform. Using the methodologies just established, three APDL topology optimizers were coded. One based on the ESO method, one based on SIMP, and the last one adding the overhang constraint in the SIMP program. In each of the programs, the material property APDL command was used to modify the elements young's modulus, therefore directly affecting its stiffness. Overall each script solves the initial FEA problem and gets the initial element values. Then it retrieves each elements sensitivity value, and uses the corresponding methodology to choose which ones to modify. Once all elements are looped through, the script re-solves the FEA and repeats until the convergence criteria are met.

\section{RESUlTS AND DISCUSSION}

\section{A. Topology Optimization - Case Study 1}

As an example, a bell crank was considered. There are two forces and a fixed hole which define the boundary conditions for this analysis. The setup in ANSYS $^{\circledR}$ is shown in Fig. 2. The element size is $2 \mathrm{~mm}$. The topology optimization results using ESO and SIMP without any additional constraints are shown in Fig. 3 and Fig. 4 at iterations 60 and 30 respectively. It can be seen that this structure may be difficult to produce using AM processes. 


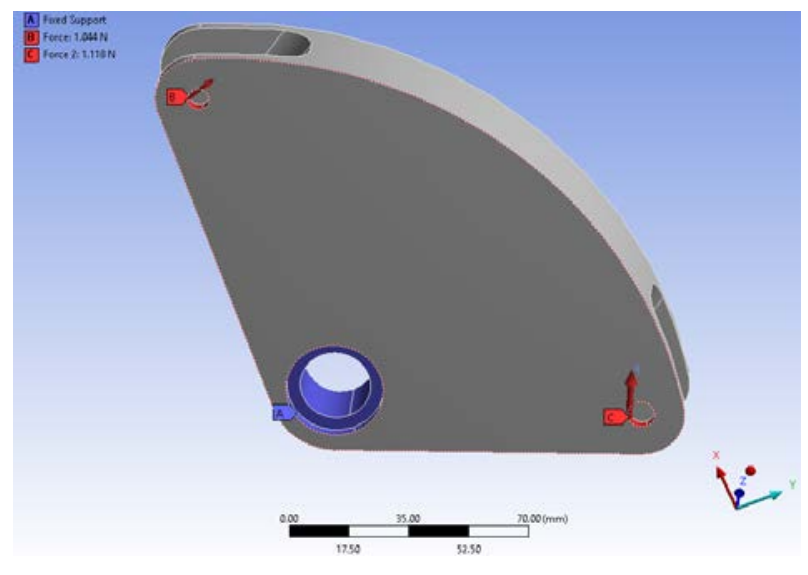

Figure 2. Case Study 1 Setup

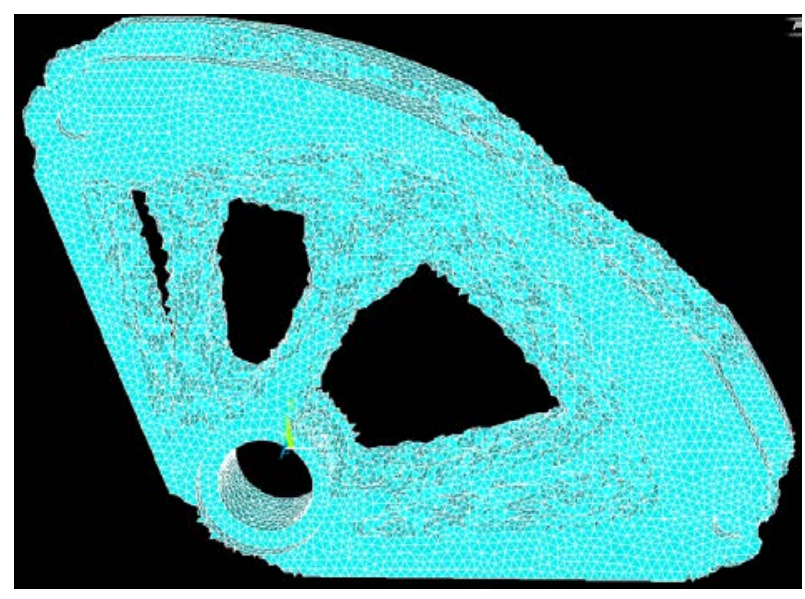

Figure 3. Case Study 1 Results after 60 Iterations using ESO

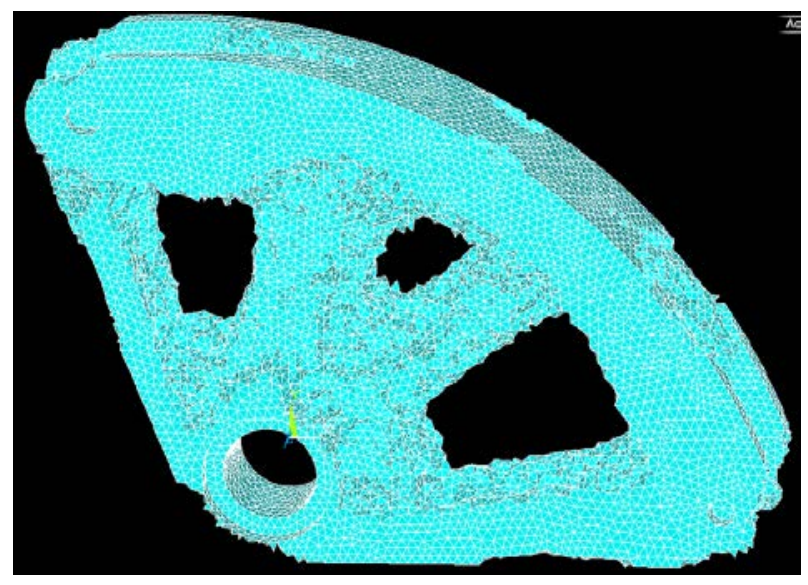

Figure 4. Case Study 1 Results after 30 Iterations using SIMP

\section{B. Topology Optimization - Case Study 2: Overhang Constraint}

Next, an MBB-beam as shown in Fig. 5 was considered. It has a fixed constraint at the bottom corner of one end and a rolling constraint at the other end, with a $1000 \mathrm{~N}$ force applied at the middle point of the top surface. It has a mesh size of 40x180. The setup in ANSYS $^{\circledR}$ is shown in Fig. 6. The SIMP method topology optimization results using half design domain with symmetry are shown in Fig. 7. The results in Fig. 8 and Fig. 9 are with the overhang constraint with varying $\delta$ values from (8). The blue line at the bottom represents the build platform, with the blue arrow specifying the build direction. By inspection it appears that this structure does indeed have less overhanging structures than the normal SIMP method.

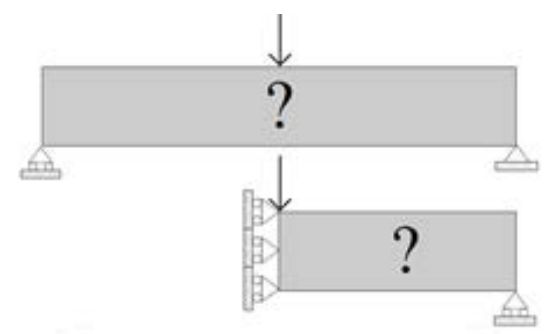

Figure 5. MBB-beam. Top: full design domain, bottom: half design domain with symmetry [7].

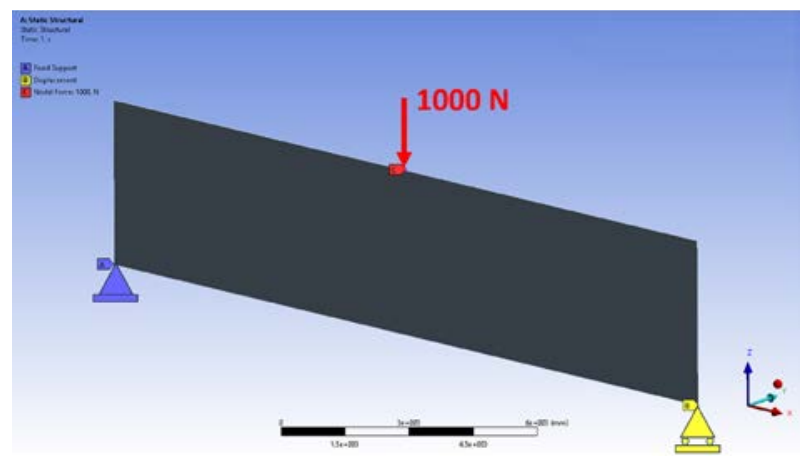

Figure 6. Case Study 2 Setup

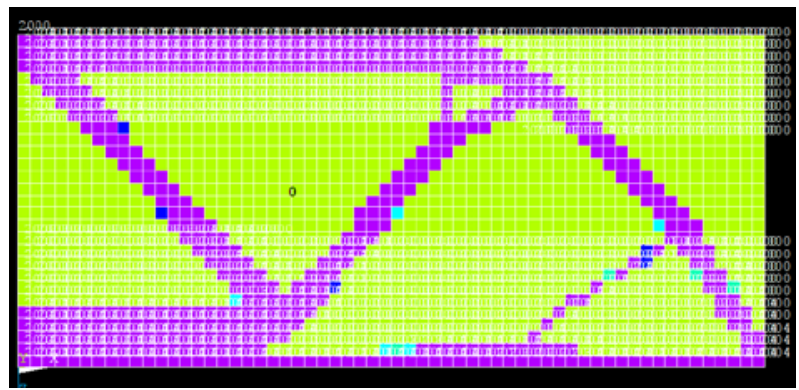

Figure 7. Case Study 2 Half Design Domain Results after 50 Iterations using SIMP (Purple elements are solid, green elements are a void)

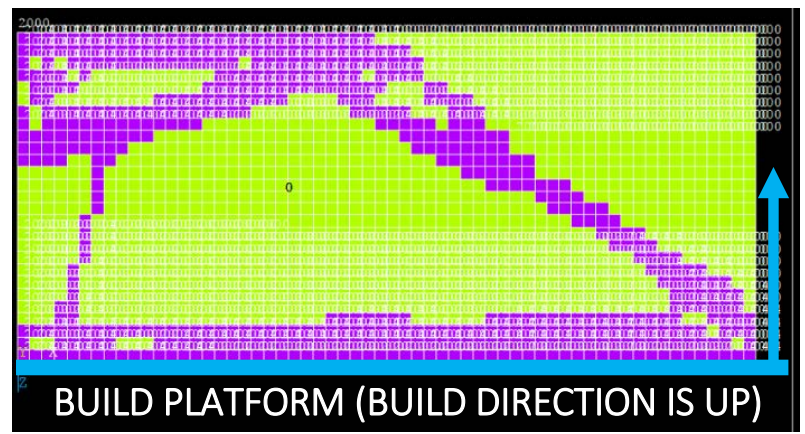

Figure 8. Case Study 2 Half Design Domain Results using Overhang Constraints, $\delta=0.00005$ (Purple elements are solid, green elements are a void) 


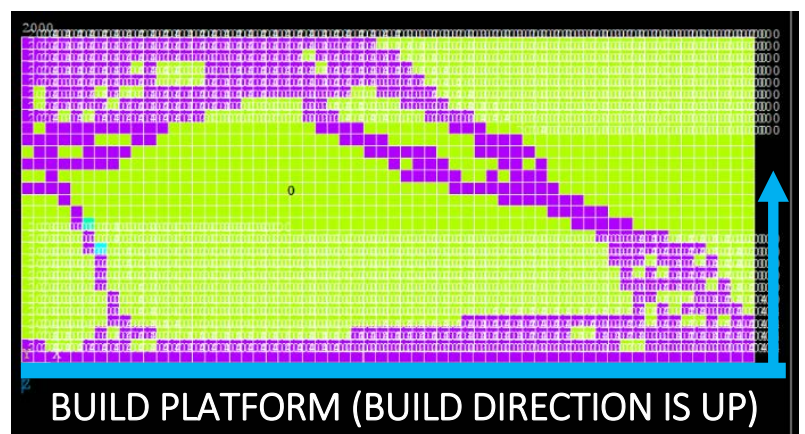

Figure 9. Case Study 2 Half Design Domain Results using Overhang Constraints, $\delta=0.0001$ (Purple elements are solid, green elements are a void)

\section{CONCLUSION}

In this paper, three topology optimization codes were developed. Two based on previous methodologies, the ESO method and the SIMP method, and the final attempted to optimize the topology for AM processes by reducing the need for support material. All were implemented in ANSYS ${ }^{\circledR}$ directly, using APDL scripts, in order to achieve a flexible and reliable development platform. The first case study looked at the implementation of the ESO and SIMP methods, and the last one looked at the implementation of the overhang constraint. The proposed topology optimization filter modifies the element sensitivities to converge the optimization towards the optimal structure that needs the least amount of material for support structures. This will reduce the manufacturing cost and time for preprocessing and post-processing operations, and reduce material waste. The established methodology can be easily developed for various additive manufacturing technologies in various industrial applications.

\section{ACKNOWLEDGMENT}

The research support provided by the Natural Science and Engineering Research Council of Canada (NSERC) and the research collaboration of SimuTech Group, Inc. are greatly appreciated.

\section{REFERENCES}

[1] X Huang and M Xie, Evolutionary topology optimization of continuum structures: methods and applications. 2010.

[2] M. K. Thompson and J. M. Thompson, ANSYS Mechanical APDL for Finite Element Analysis. Butterworth-Heinemann, 2017.

[3] A. Lalehpour and A. Barari, "Post processing for Fused Deposition Modeling Parts with Acetone Vapour Bath," IFAC-Pap., vol. 49, no. 31, pp. 42-48, 2016.
[4] J. Vanek, J. A. G. Galicia, and B. Benes, “Clever support: Efficient support structure generation for digital fabrication,” in Computer graphics forum, 2014, vol. 33, pp. 117-125.

[5] M. Langelaar, "An additive manufacturing filter for topology optimization of print-ready designs," Struct. Multidiscip. Optim., vol. 55, no. 3, pp. 871-883, Mar. 2017.

[6] M. P. Bendsøe and O. Sigmund, "Topology optimization by distribution of isotropic material," in Topology Optimization, Springer, Berlin, Heidelberg, 2004, pp. 169.

[7] O. Sigmund, "A 99 line topology optimization code written in Matlab,” Struct. Multidiscip. Optim., vol. 21, no. 2, pp. 120-127, 2001.

[8] A. Barari, "Inspection of the machined surfaces using manufacturing data,” J. Manuf. Syst., vol. 32, no. 1, pp. 107-113, 2013.

[9] F. Kaji and A. Barari, "Evaluation of the surface roughness of additive manufacturing parts based on the modelling of cusp geometry,” IFAC-Pap., vol. 48, no. 3, pp. 658-663, 2015.

[10] A. Barari, H. A. Kishawy, F. Kaji, and M. A. Elbestawi, "On the surface quality of additive manufactured parts," Int. J. Adv. Manuf. Technol., vol. 89, no. 5-8, pp. 19691974, 2017.

[11] S. Sikder, A. Barari, and H. A. Kishawy, "Effect of adaptive slicing on surface integrity in additive manufacturing," in ASME 2014 International Design Engineering Technical Conferences and Computers and Information in Engineering Conference, 2014, p. V01AT02A052-V01AT02A052.

[12] H. Gohari, A. Barari, and H. Kishawy, "Using Multistep Methods in Slicing $2 \$ 1 / 2 \$$ Dimensional Parametric Surfaces for Additive Manufacturing Applications,” IFAC-Pap., vol. 49, no. 31, pp. 67-72, 2016.

[13] H. Gohari and A. Barari, “A quick deviation zone fitting in coordinate metrology of NURBS surfaces using principle component analysis,” Measurement, vol. 92, pp. 352-364, 2016.

[14] S. Moaveni, Finite element analysis theory and application with ANSYS, 3/e. Pearson Education India, 2011.

[15] C. S. Jog, "Topology design of structures using a dual algorithm and a constraint on the perimeter,” Int. J. Numer. Methods Eng., vol. 54, no. 7, pp. 1007-1019, 2002. 